\title{
QUALIDADE FISIOLÓGICA DE SEMENTES DE SOJA TRATADAS COM FUNGICIDAS DURANTE E APÓS O ARMAZENAMENTO ${ }^{1}$
}

\author{
NÁDIAGRACIELE KROHN² ${ }^{2}$ MARLENE DE MATOS MALAVASI ${ }^{3}$
}

\begin{abstract}
RESUMO - O presente trabalho teve o objetivo de avaliar o efeito do tratamento fungicida durante e após a armazenagem, na qualidade fisiológica de sementes de soja. Utilizaram-se sementes da cultivar BR-16, produzidas na safra de 1999/2000 e armazenadas em Marechal Cândido Rondon PR. Para o tratamento utilizou-se a mistura de carbendazin + thiram, na dosage de $30 \mathrm{e} 70 \mathrm{~g}$ de i.a./ $100 \mathrm{~kg}$ de semente, respectivamente. Realizou-se o tratamento de amostras mensalmente, de maio a dezembro de 2000, sendo as sementes analisadas imediatamente após o procedimento. Sementes sem tratamento representaram a testemunha. As amostras tratadas e armazenadas foram analisadas e comparadas com as tratadas e analisadas imediatamente antes da semeadura (dezembro de 2000). A qualidade das sementes foi avaliada através dos testes de germinação, de tetrazólio para vigor e viabilidade e emergência em campo. $O$ delineamento experimental foi inteiramente casualizado, com quatro repetições por tratamento para cada teste. Realizou-se a comparação das médias através do teste de Tukey a 5\% de probabilidade. O teste de germinação e o de tetrazólio não evidenciaram efeito negativo do tratamento fungicida anterior ao período de armazenagem sobre a qualidade das sementes. As sementes tratadas e não apresentaram decréscimo de viabilidade e de vigor, atingindo valores de germinação inferiores a $75 \%$, em dezembro de 2000 . Na emergência em campo, constatou-se que as sementes mantidas tratadas por mais de quatro meses apresentaram desempenho inferior comparativamente às tratadas nas demais épocas.
\end{abstract}

Termos para indexação: Glycine max, conservação de sementes, viabilidade, vigor.

\section{PHYSIOLOGICAL QUALITY OF SOYBEAN SEEDS TREATED WITH FUNGICIDE DURING ANDAFTER STORAGE}

ABSTRACT - The present research evaluated the chemical treatment effect before the storage of
soybean seeds on their physiological quality. Seeds from the cultivar BR-16, produced and stored
in Marechal Cândido Rondon-PR, 1999/2000, were utilized. For treatment the mixture of carbendazin
+ thiram, at the dosage 30 and $70 \mathrm{~g}$ of active ingredient $/ 100 \mathrm{~kg}^{-1}$ of seed, respectively, was utilized.
Seeds were treated and analyzed monthly from May to December. Seeds without treatment
represented the control. In December all the samples previously treated and stored were analyzed
and compared with the seeds treated and analyzed at the sowing time (December). The seed
quality was evaluated by the standard germination test, tetrazolium test for vigor and viability and
field emergence. A completely randomized experimental design was employed, with four replications
per treatment, for every test. The means were compared by the Tukey test at the probability level
of $5 \%$. The standard germination test and the tetrazolium test did not show negative effect of the
previous chemical treatment on the quality of the seeds. But treated and non treated seeds

${ }^{1}$ Submetido em 06/10/2003. Aceito para publicação em 16/06/2004.

${ }^{2}$ Eng $^{\mathrm{a}}$ Agr $^{\mathrm{a}}$, Pós-Graduanda em Agronomia - Sistemas de Produção, FEIS/ UNESP, Av Brasil, Centro, 56 - Caixa Postal 31, CEP 15385-000, Ilha Solteira-SP, e-mail: nadiakrohn@yahoo.com.br.

\footnotetext{
${ }^{3}$ Eng $^{\mathrm{a}}$. Agr ${ }^{\mathrm{a}}$, Prof ${ }^{\mathrm{a}}$ PhD Efetiva do Curso de Agronomia, UNIOESTE, Rua Pernambuco, 1777, CEP: 85960-000, Marechal Cândido Rondon-PR, email:malavasi@unioeste.br.
} 
presented decrease in viability and vigor, reaching a value inferior to $75 \%$, in December. In the field emergence test, it was observed that the seeds that were treated for more than four months showed lower performance than the seeds that were treated for shorter periods.

Index terms: Glycine max, seed conservation, viability, vigor.

\section{INTRODUÇÃO}

O sucesso de uma lavoura está condicionado à utilização de sementes de alta qualidade. Neste sentido, $o$ armazenamento constitui uma etapa onde se deve procurar reduzir ao mínimo a velocidade e a intensidade do processo de deterioração, principalmente no caso da soja, pois geralmente, inicia-se a colheita em fevereiro e as sementes são armazenadas por um período de oito meses, até a semeadura.

No período de desenvolvimento e maturação, a soja pode ser atacada por diversos patógenos, entre estes se destacam Phomopsis spp., Fusarium spp., Colletotrichum truncatum e Cercospora kikuchii, que tem potencial para causar prejuízos na qualidade das sementes. No entanto, esses fungos diminuem sua incidência quando as sementes são armazenadas (Goulart, 1997; Goulart et al., 1999).

$\mathrm{Na}$ armazenagem as sementes podem ser afetadas por fungos como Aspergillus spp. e Penicillium spp., os quais contribuem para a sua deterioração (França Neto \& Henning, 1984; Dhingra, 1985; Henning, 1987; Novembre \& Marcos Filho, 1991; Goulart, 1997; Goulart et al., 1999).

Uma técnica que pode ser utilizada para diminuir a ação desses patógenos que se associam às sementes, nas diversas fases do processo de produção é o tratamento com fungicidas. O considerado tem grande importância para a cultura em questão, devido à expansão da área cultivada para as regiões Centro-Oeste e Norte (Embrapa, 1999), evitando a introdução de doenças nessas áreas, e a reintrodução em áreas já usadas para a cultura (Goulart, 1998). O tratamento pode garantir também bom estande de plantas quando a semeadura é realizada em condições desfavoráveis de temperatura e disponibilidade hídrica. De acordo com Pereira et al. (1993), o fungicida pode propiciar proteção às sementes, quando semeadas em condições adversas, por um período de 4 a 12 dias, dependendo do vigor das mesmas. Além do mais, o uso de substâncias químicas em sementes, antes do armazenamento, poderia ter algum efeito protetor, por eliminar patógenos, principalmente os fungos de campo e de armazenamento.

Nas culturas do feijão (Novembre \& Marcos Filho, 1991), do milho (Oliveira et al., 1997) e do amendoim (Tella et al., 1976) a eficiência do tratamento fungicida antecipado ao armazenamento está comprovada. Porém, não existe unanimidade quanto à eficiência desta técnica, para sementes de soja.

Diversos autores como Marcos Filho \& Souza (1983), Lucca Filho et al. (1983a), Lucca Filho et al. (1983b), Henning \& Zorato (1997), Zorato (1998), Goulart et al. (1999) e Zorato \& Henning (2001) demonstraram ser viável o tratamento fungicida de sementes de soja antecipado, por não ter ocorrido efeito negativo dessa prática na qualidade das sementes durante e após o armazenamento e, de modo geral, houve melhor conservação das sementes tratadas durante a armazenagem em comparação às não tratadas. No entanto, Goulart \& Cassetari Neto (1987), Carvalho \& Jacinto (1979) e França Neto \& Henning (1984) não evidenciaram resposta positiva quanto à utilização desta técnica, sendo que recomendam que o tratamento seja feito imediatamente antes da semeadura.

$\mathrm{O}$ tratamento fungicida antecipado ao armazenamento traz o inconveniente de as sementes tratadas não poderem ser comercializadas e destinadas à industria, principalmente no Brasil, onde a incerteza quanto ao comportamento do mercado de sementes é muito grande (Goulart et al., 1999).

De acordo com o comentado, realizou-se o presente trabalho para verificar o efeito do tratamento fungicida durante e após o armazenamento, na qualidade fisiológica de sementes de soja.

\section{MATERIALE MÉTODOS}

Este estudo foi conduzido no Laboratório de Tecnologia de Sementes da Unioeste, em Marechal Cândido Rondon, PR. Utilizaram-se sementes de soja (Glycine max L.), cultivar BR16 , provenientes de uma amostra do lote comercial, produzidas na safra de 1999/2000.

O trabalho teve início em maio de 2000. A amostra foi subdividida, sendo que as sementes receberam tratamento fungicida em diferentes épocas $210,180,150,120,90,30$ dias antes e na época de semeadura considerada como dezembro, perfazendo oito épocas de tratamento e quinze épocas de análises, descritas a seguir: $\mathbf{E}_{\mathbf{1}} \mathbf{T}$ - sementes tratadas e analisadas em maio, 210 dias antes da semeadura; $\mathbf{E}_{2} \mathbf{T}$ sementes tratadas e analisadas em junho, 180 dias antes da semeadura; $\mathbf{E} \mathbf{3}$ T- sementes tratadas e analisadas em julho, 150 
dias antes da semeadura; $\mathbf{E}_{\mathbf{4}} \mathbf{T}$ - sementes tratadas e analisadas em agosto, 120 dias antes da semeadura; $\mathbf{E}_{\mathbf{5}} \mathbf{T}$ - sementes tratadas e analisadas em setembro, 90 dias antes da semeadura; $\mathbf{E}_{\mathbf{6}} \mathbf{T}$ - sementes tratadas e analisadas em outubro, 60 dias antes da semeadura; $\mathbf{E}_{7} \mathbf{T}$ - sementes tratadas e analisadas em novembro, 30 dias antes da semeadura; $\mathbf{E}_{\mathbf{8}} \mathbf{T}$ - sementes tratadas e analisadas em dezembro; $\mathbf{E}_{\mathbf{1}} \mathbf{A}$ - sementes analisadas em dezembro, 210 dias após tratamento (maio) e armazenamento; $\mathbf{E}_{2} \mathbf{A}$ - sementes analisadas em dezembro, 180 dias após tratamento (junho) e armazenamento; $\mathbf{E}_{\mathbf{3}} \mathbf{A}$ - sementes analisadas em dezembro, 150 dias após tratamento (julho) e armazenamento; $\mathbf{E}_{\mathbf{4}} \mathbf{A}$ - sementes analisadas em dezembro, 120 dias após tratamento (agosto) e armazenamento; $\mathbf{E}_{\mathbf{5}} \mathbf{A}$ sementes analisadas em dezembro, 90 dias após tratamento (setembro) e armazenamento; $\mathbf{E}_{6} \mathbf{A}$ - sementes analisadas em dezembro, 60 dias após tratamento (outubro) e armazenamento; $\mathbf{E}_{7} \mathbf{A}$ - sementes analisadas em dezembro, 30 dias após tratamento (novembro) e armazenamento.

O tratamento fungicida constituiu-se da aplicação da mistura carbendazin $(30 \mathrm{~g}$ de ingrediente ativo $/ 100 \mathrm{~kg}$ de sementes $)+$ thiram $(70 \mathrm{~g}$ de ingrediente ativo $/ 100 \mathrm{~kg}$ de sementes), sendo que a esta mistura adicionou-se $100 \mathrm{ml}$ de água/100kg de sementes. As sementes foram colocadas em sacos plásticos, adicionaram-se os produtos e agitou-se até a distribuição uniforme sobre as mesmas. A testemunha constituiu-se de sementes sem tratamento. Após o tratamento, as sementes foram acondicionadas em bandejas plásticas e assim permaneceram até que ocorresse a secagem dos produtos aplicados. As sementes tratadas e a testemunha ficaram armazenadas em sacos de papel, em ambiente cuja temperatura variou de $20-25^{\circ} \mathrm{C}$.

A qualidade das sementes foi avaliada através dos seguintes testes:

Germinação - conduzido de acordo com as Regras para Análise de Sementes (Brasil, 1992), com 400 sementes por tratamento, distribuídas em quatro repetições de 100 sementes, utilizando como substrato o papel germitest, autoclavado a $120^{\circ} \mathrm{C}$ por vinte minutos, umedecido em água na proporção de 2,5 vezes o peso do papel e colocados em temperatura constante de $25^{\circ} \mathrm{C}$; a avaliação foi realizada ao quinto e oitavo dia após a semeadura.

Teste de Tetrazólio - utilizou-se a metodologia descrita por França Neto et al. (1998), conduzida com 100 sementes por tratamento, distribuídas em quatro repetições de 25 sementes, pré-condicionadas em papel umedecido, e mantidas nestas condições por dezesseis horas a $25^{\circ} \mathrm{C}$, para a remoção do tegumento; após as sementes foram colocadas em recipientes plásticos, cobertas com a solução do sal de tetrazólio $(0,075 \%)$ e mantidas em câmara escura a $40^{\circ} \mathrm{C}$ por três horas. Passado este tempo, removeu-se a solução de tetrazólio e lavaram-se as sementes com água. Realizou-se a interpretação logo após, cortando-se as sementes longitudinalmente através do seu eixo embrionário. Analisaram-se as superfícies interna e externa dos cotilédones, anotando-se os valores de viabilidade, vigor e danos por umidade.

Grau de Umidade - determinado pelo método da estufa a $105^{\circ} \pm 3^{\circ} \mathrm{C}$ (Brasil, 1992), utilizando-se quatro repetições de $4 \mathrm{~g}$ de sementes por tratamento.

Emergência em Campo - realizada de outubro a dezembro de 2000, dentro da época recomendada para a semeadura da cultura, na região oeste do Paraná. Utilizaramse 400 sementes por tratamento, distribuídas em quatro repetições de 100 sementes, semeadas em sulcos de $1,00 \mathrm{~m}$ de comprimento, espaçadas de $0,15 \mathrm{~m}$ e à profundidade aproximada de $0,03 \mathrm{~m}$. As plântulas foram contadas no décimo quinto dia após a semeadura.

Procedimentos Estatísticos - foi empregado o delineamento experimental inteiramente casualizado. Todos os testes foram conduzidos com quatro repetições e para a comparação entre as médias foi utilizado o teste de Tukey, a $5 \%$ de probabilidade. Os dados porcentuais sofreram transformação em arco seno $(\% / 100)^{0,5}$. Na execução da análise foi utilizado o "Sistema de Análise Estatística para Microcomputadores - SANEST".

\section{RESULTADOS E DISCUSSÃO}

Determinou-se o grau de umidade das sementes para acompanhar as condições de armazenagem, sendo que não foi aplicada análise estatística para os valores. Os resultados encontram-se na Tabela 1. Verificou-se que a variação foi baixa, tanto para sementes tratadas como para a testemunha.

A viabilidade medida pelo teste de germinação (Tabela 2) decresceu consideravelmente na época $\mathrm{E}_{6} \mathrm{~T}$ (sementes tratadas e analisadas em outubro, 60 dias antes da semeadura), tanto para as sementes tratadas como para a testemunha. Constatouse que as sementes analisadas em dezembro, independentemente do tratamento e da época de realização, apresentaram viabilidade inferior a $80 \%$, fato que impede a comercialização do lote como sementes certificadas, de acordo com a legislação vigente (Brasil, 1993). 
TABELA 1. Grau de umidade (\%) de sementes de soja da cultivar BR-16, produzidas na safra de 1999/2000, tratadas com fungicida em diferentes épocas, e armazenadas em Marechal Cândido Rondon, PR.

\begin{tabular}{crc}
\hline & \multicolumn{2}{c}{ Tratamento } \\
\cline { 2 - 3 } Época & \multicolumn{1}{c}{$\mathrm{T}_{0}$} & $\mathrm{~T}_{1}$ \\
\hline $\mathrm{E}_{1} \mathrm{~T}$ & 11,4 & 11,4 \\
$\mathrm{E}_{2} \mathrm{~T}$ & 10,6 & 10,3 \\
$\mathrm{E}_{3} \mathrm{~T}$ & 9,7 & 10,9 \\
$\mathrm{E}_{4} \mathrm{~T}$ & 9,3 & 10,0 \\
$\mathrm{E}_{5} \mathrm{~T}$ & 10,0 & 10,2 \\
$\mathrm{E}_{6} \mathrm{~T}$ & 10,6 & 11,2 \\
$\mathrm{E}_{7} \mathrm{~T}$ & 9,7 & 10,7 \\
$\mathrm{E}_{8} \mathrm{~T}$ & 10,3 & 10,2 \\
$\mathrm{E}_{1} \mathrm{~A}$ & 10,3 & 10,3 \\
$\mathrm{E}_{2} \mathrm{~A}$ & 10,3 & 10,2 \\
$\mathrm{E}_{3} \mathrm{~A}$ & 10,3 & 10,1 \\
$\mathrm{E}_{4} \mathrm{~A}$ & 10,3 & 10,3 \\
$\mathrm{E}_{5} \mathrm{~A}$ & 10,3 & 10,2 \\
$\mathrm{E}_{6} \mathrm{~A}$ & 10,3 & 10,0 \\
$\mathrm{E}_{7} \mathrm{~A}$ & 10,3 & 10,4 \\
\hline
\end{tabular}

$\mathrm{T}_{1}$ - sementes tratadas com Carbendazin + Thiram; $\mathrm{T}_{0}-$ sementes sem tratamento.

TABELA 2. Influência do tratamento fungicida, realizado em diferentes épocas, sobre a viabilidade (\%), avaliada pelo teste de germinação, em sementes de soja da cultivar BR-16, produzidas e armazenadas na safra de 1999/2000, em Marechal Cândido Rondon, PR.

\begin{tabular}{llll}
\hline & \multicolumn{2}{c}{ Tratamento } & \\
\cline { 2 - 3 } Época & \multicolumn{1}{c}{$\mathrm{T}_{0}$} & $\mathrm{~T}_{1}$ & $\mathrm{CV}(\%)$ \\
\hline $\mathrm{E}_{1} \mathrm{~T}$ & $95 \mathrm{Aabc}$ & $96 \mathrm{Aa}$ & 4,03 \\
$\mathrm{E}_{2} \mathrm{~T}$ & $95 \mathrm{Aab}$ & $92 \mathrm{Aab}$ & 4,29 \\
$\mathrm{E}_{3} \mathrm{~T}$ & $91 \mathrm{Bbcd}$ & $97 \mathrm{Aa}$ & 3,81 \\
$\mathrm{E}_{4} \mathrm{~T}$ & $95 \mathrm{Aab}$ & $93 \mathrm{Aab}$ & 5,24 \\
$\mathrm{E}_{5} \mathrm{~T}$ & $97 \mathrm{Aa}$ & $95 \mathrm{Aa}$ & 2,46 \\
$\mathrm{E}_{6} \mathrm{~T}$ & $89 \mathrm{Acd}$ & $84 \mathrm{Abc}$ & 3,96 \\
$\mathrm{E}_{7} \mathrm{~T}$ & $86 \mathrm{Bd}$ & $92 \mathrm{Aab}$ & 2,79 \\
$\mathrm{E}_{8} \mathrm{~T}$ & $67 \mathrm{Ae}$ & $80 \mathrm{Acd}$ & 3,87 \\
$\mathrm{E}_{1} \mathrm{~A}$ & $67 \mathrm{Ae}$ & $74 \mathrm{Acd}$ & 5,49 \\
$\mathrm{E}_{2} \mathrm{~A}$ & $67 \mathrm{Be}$ & $73 \mathrm{Acd}$ & 5,69 \\
$\mathrm{E}_{3} \mathrm{~A}$ & $67 \mathrm{Ae}$ & $75 \mathrm{Acd}$ & 3,34 \\
$\mathrm{E}_{4} \mathrm{~A}$ & $67 \mathrm{Be}$ & $71 \mathrm{Ad}$ & 4,39 \\
$\mathrm{E}_{5} \mathrm{~A}$ & $67 \mathrm{Be}$ & $75 \mathrm{Acd}$ & 5,00 \\
$\mathrm{E}_{6} \mathrm{~A}$ & $67 \mathrm{Ae}$ & $76 \mathrm{Acd}$ & 4,48 \\
$\mathrm{E}_{7} \mathrm{~A}$ & $67 \mathrm{Be}$ & $73 \mathrm{Acd}$ & 6,19 \\
\hline $\mathrm{CV}(\%)$ & 3,83 & 4,81 & \\
\hline
\end{tabular}

$\mathrm{T}_{1}$ - sementes tratadas com Carbendazin + Thiram; $\mathrm{T}_{0}-$ sementes sem tratamento. Médias seguidas pela mesma letra maiúscula na linha e minúscula na coluna, não diferem entre si pelo teste de Tukey, a 5\% de probabilidade.
Ainda de acordo com a Tabela 2, não se constatou efeito negativo do tratamento fungicida anterior ao armazenamento, pois na maioria das épocas a testemunha e as sementes tratadas apresentaram o mesmo desempenho, e em alguns casos as últimas apresentaram desempenho superior.

Esses resultados são evidenciados na emergência em campo (Tabela 3), onde na maioria das vezes sementes tratadas apresentaram desempenho superior à testemunha. Segundo Zorato (1998), o teste de germinação é realizado em condições ideais de temperatura e umidade ao contrário da emergência em campo. Conforme Goulart (1998), a semente de soja inicia o processo de germinação e emerge rapidamente em solos com boa disponibilidade hídrica e temperatura adequada. Quando estas condições não são satisfeitas, o processo anteriormente citado é mais lento, proporcionando aos fungos do solo e da própria semente maior oportunidade de ataque para deteriorar as sementes ou causar a morte das plântulas. Dessa forma explica-se a diferença entre os resultados obtidos no teste de germinação e na emergência em campo.

Verificou-se também que as sementes mantidas tratadas por período superior a quatro meses apresentaram emergência inferior ( $\mathrm{E}_{1} \mathrm{~A}, \mathrm{E}_{2} \mathrm{~A}$ e $\left.\mathrm{E}_{3} \mathrm{~A}\right)$ em dezembro, comparativamente às demais épocas de tratamento. Provavelmente houve algum efeito fitotóxico dos fungicidas. Goulart et al. (1999) comprovaram redução da qualidade fisiológica de sementes tratadas com carbendazin isolado e armazenadas por período superior a 60 dias. Quando o fungicida foi misturado com

TABELA 3. Influência do tratamento fungicida, realizado em diferentes épocas, sobre o vigor (\%), avaliado pela emergência em campo, em sementes de soja da cultivar BR-16, produzidas e armazenadas na safra de 1999/2000, em Marechal Cândido Rondon, PR.

\begin{tabular}{cccc}
\hline & \multicolumn{2}{c}{ Tratamento } & \\
\cline { 2 - 3 } Época & $\mathrm{T}_{0}$ & \multicolumn{1}{c}{$\mathrm{T}_{1}$} & $\mathrm{CV}(\%)$ \\
\hline $\mathrm{E}_{6} \mathrm{~T}$ & $91 \mathrm{Ba}$ & $95 \mathrm{Aa}$ & 3,22 \\
$\mathrm{E}_{7} \mathrm{~T}$ & $80 \mathrm{Bb}$ & $91 \mathrm{Aabc}$ & 6,41 \\
$\mathrm{E}_{8} \mathrm{~T}$ & $78 \mathrm{Ab}$ & $84 \mathrm{Abcd}$ & 4,41 \\
$\mathrm{E}_{1} \mathrm{~A}$ & $78 \mathrm{Ab}$ & $84 \mathrm{Acd}$ & 3,61 \\
$\mathrm{E}_{2} \mathrm{~A}$ & $78 \mathrm{Bb}$ & $81 \mathrm{Ad}$ & 3,84 \\
$\mathrm{E}_{3} \mathrm{~A}$ & $78 \mathrm{Bb}$ & $88 \mathrm{Abcd}$ & 3,53 \\
$\mathrm{E}_{4} \mathrm{~A}$ & $78 \mathrm{Bb}$ & $90 \mathrm{Aabcd}$ & 3,75 \\
$\mathrm{E}_{5} \mathrm{~A}$ & $78 \mathrm{Bb}$ & $91 \mathrm{Aabc}$ & 2,55 \\
$\mathrm{E}_{6} \mathrm{~A}$ & $78 \mathrm{Bb}$ & $91 \mathrm{Aabc}$ & 2,71 \\
$\mathrm{E}_{7} \mathrm{~A}$ & $78 \mathrm{Ab}$ & $92 \mathrm{Aab}$ & 4,39 \\
\hline $\mathrm{CV}(\%)$ & 4,21 & 4,17 & \\
\hline
\end{tabular}

$\mathrm{T}_{1}$ - sementes tratadas com Carbendazin + Thiram; $\mathrm{T}_{0}-$ sementes sem tratamento. Médias seguidas pela mesma letra maiúscula na linha e minúscula na coluna, não diferem entre si pelo teste de Tukey, a 5\% de probabilidade. 
tolyfluanid, o efeito fitotóxico reduziu-se significativamente. Ainda de acordo com os autores, a queda na qualidade fisiológica foi visualizada principalmente na emergência em campo, quando comparada com os testes realizados em laboratório.

Analisando-se a viabilidade (Tabela 4) e o vigor (Tabela 5), avaliados através do teste de tetrazólio, nota-se queda destes atributos fisiológicos na época $\mathrm{E}_{7} \mathrm{~T}$ (sementes tratadas e analisadas em novembro, 30 dias antes da semeadura) tanto para as sementes tratadas como para a testemunha. Dessa forma, comprova-se a afirmação de Menten (1991) ao considerar que o vigor das sementes influi na resposta ao tratamento fungicida. Observou-se que o atributo vigor apresentou redução mais drástica que a viabilidade. De acordo com Delouche e Baskin (1973), a perda do poder germinativo é, provavelmente, a conseqüência final do processo deteriorativo, pois ocorrem alterações anteriores como a redução da resistência às condições adversas do ambiente (redução do vigor).

O teste de tetrazólio raras vezes mostrou diferença entre as sementes tratadas e a testemunha, tanto para a viabilidade (Tabela 4) como para o vigor (Tabela 5), ao contrário da

TABELA 4. Influência do tratamento fungicida, realizado em diferentes épocas, sobre a viabilidade (\%), avaliada pelo teste de tetrazólio, em sementes de soja da cultivar BR-16, produzidas e armazenadas na safra de 1999/2000, em Marechal Cândido Rondon, PR.

\begin{tabular}{|c|c|c|c|}
\hline \multirow[b]{2}{*}{ Época } & \multicolumn{2}{|c|}{ Tratamento } & \multirow[b]{2}{*}{$\mathrm{CV}(\%)$} \\
\hline & $\mathrm{T}_{0}$ & $\mathrm{~T}_{1}$ & \\
\hline $\mathrm{E}_{1} \mathrm{~T}$ & $97 \mathrm{Aab}$ & $97 \mathrm{Aa}$ & 8,71 \\
\hline $\mathrm{E}_{2} \mathrm{~T}$ & $94 \mathrm{Aab}$ & $92 \mathrm{Aab}$ & 4,30 \\
\hline $\mathrm{E}_{3} \mathrm{~T}$ & $99 \mathrm{Aa}$ & 96 Aabc & 8,86 \\
\hline $\mathrm{E}_{4} \mathrm{~T}$ & $97 \mathrm{Aab}$ & 90 Aabc & 8,07 \\
\hline $\mathrm{E}_{5} \mathrm{~T}$ & $87 \mathrm{Abc}$ & 86 Aabc & 4,80 \\
\hline $\mathrm{E}_{6} \mathrm{~T}$ & $96 \mathrm{Aab}$ & $84 \mathrm{Aabc}$ & 9,73 \\
\hline $\mathrm{E}_{7} \mathrm{~T}$ & $88 \mathrm{Abc}$ & $82 \mathrm{Abc}$ & 7,60 \\
\hline $\mathrm{E}_{8} \mathrm{~T}$ & $74 \mathrm{Ac}$ & $77 \mathrm{Ac}$ & 4,29 \\
\hline $\mathrm{E}_{1} \mathrm{~A}$ & $74 \mathrm{Ac}$ & $77 \mathrm{Ac}$ & 4,29 \\
\hline $\mathrm{E}_{2} \mathrm{~A}$ & $74 \mathrm{Bc}$ & $78 \mathrm{Ac}$ & 10,71 \\
\hline $\mathrm{E}_{3} \mathrm{~A}$ & $74 \mathrm{Ac}$ & $87 \mathrm{Aabc}$ & 6,76 \\
\hline $\mathrm{E}_{4} \mathrm{~A}$ & $74 \mathrm{Ac}$ & $80 \mathrm{Ac}$ & 4,75 \\
\hline $\mathrm{E}_{5} \mathrm{~A}$ & $74 \mathrm{Ac}$ & $86 \mathrm{Aabc}$ & 9,53 \\
\hline $\mathrm{E}_{6} \mathrm{~A}$ & $74 \mathrm{Ac}$ & $81 \mathrm{Abc}$ & 5,86 \\
\hline $\mathrm{E}_{7} \mathrm{~A}$ & $74 \mathrm{Ac}$ & $78 \mathrm{Ac}$ & 8,74 \\
\hline $\mathrm{CV}(\%)$ & 8,12 & 7,99 & \\
\hline
\end{tabular}

$\mathrm{T}_{1}$ - sementes tratadas com Carbendazin + Thiram; $\mathrm{T}_{0}-$ sementes sem tratamento. Médias seguidas pela mesma letra maiúscula na linha e minúscula na coluna, não diferem entre si pelo teste de Tukey, a 5\% de probabilidade. emergência em campo (Tabela 3). Segundo França Neto et al. (1998) uma das limitações do teste em questão é não mostrar a eficácia de tratamentos químicos, nem os efeitos prejudiciais que estes possam causar.

TABELA 5. Influência do tratamento fungicida, realizado em diferentes épocas, sobre a vigor (\%), avaliado pelo teste de tetrazólio, em sementes de soja da cultivar BR-16, produzidas e armazenadas na safra de 1999/2000, em Marechal Cândido Rondon, PR.

\begin{tabular}{clll}
\hline & \multicolumn{2}{c}{ Tratamento } & \\
\cline { 2 - 3 } Época & \multicolumn{1}{c}{$\mathrm{T}_{0}$} & \multicolumn{1}{c}{$\mathrm{T}_{1}$} & $\mathrm{CV}(\%)$ \\
\hline $\mathrm{E}_{1} \mathrm{~T}$ & $90 \mathrm{Aab}$ & $92 \mathrm{Aa}$ & 4,10 \\
$\mathrm{E}_{2} \mathrm{~T}$ & $91 \mathrm{Aab}$ & $90 \mathrm{Aa}$ & 6,47 \\
$\mathrm{E}_{3} \mathrm{~T}$ & $88 \mathrm{Aab}$ & $89 \mathrm{Aab}$ & 6,02 \\
$\mathrm{E}_{4} \mathrm{~T}$ & $94 \mathrm{Aa}$ & $84 \mathrm{Babc}$ & 5,50 \\
$\mathrm{E}_{5} \mathrm{~T}$ & $83 \mathrm{Abc}$ & $83 \mathrm{Aabc}$ & 4,56 \\
$\mathrm{E}_{6} \mathrm{~T}$ & $85 \mathrm{Aabc}$ & $79 \mathrm{Aabcd}$ & 7,33 \\
$\mathrm{E}_{7} \mathrm{~T}$ & $76 \mathrm{Ac}$ & $81 \mathrm{Aabcd}$ & 6,13 \\
$\mathrm{E}_{8} \mathrm{~T}$ & $45 \mathrm{Ad}$ & $52 \mathrm{Ae}$ & 11,48 \\
$\mathrm{E}_{1} \mathrm{~A}$ & $45 \mathrm{Ad}$ & $55 \mathrm{Ae}$ & 8,26 \\
$\mathrm{E}_{2} \mathrm{~A}$ & $45 \mathrm{Bd}$ & $61 \mathrm{Ade}$ & 12,20 \\
$\mathrm{E}_{3} \mathrm{~A}$ & $45 \mathrm{Ad}$ & $67 \mathrm{Abcde}$ & 11,11 \\
$\mathrm{E}_{4} \mathrm{~A}$ & $45 \mathrm{Bd}$ & $57 \mathrm{Ae}$ & 12,40 \\
$\mathrm{E}_{5} \mathrm{~A}$ & $45 \mathrm{Bd}$ & $62 \mathrm{Ade}$ & 9,94 \\
$\mathrm{E}_{6} \mathrm{~A}$ & $45 \mathrm{Ad}$ & $66 \mathrm{Acde}$ & 13,05 \\
$\mathrm{E}_{7} \mathrm{~A}$ & $45 \mathrm{Ad}$ & $67 \mathrm{Abcde}$ & 15,67 \\
\hline $\mathrm{CV}(\%)$ & 5,96 & 9,04 & \\
\hline
\end{tabular}

$\mathrm{T}_{1}$ - sementes tratadas com Carbendazin + Thiram; $\mathrm{T}_{0}-$ sementes sem tratamento. Médias seguidas pela mesma letra maiúscula na linha e minúscula na coluna, não diferem entre si pelo teste de Tukey, a 5\% de probabilidade.

Verifica-se pela Tabela 6 que a redução do vigor e da viabilidade pode ser atribuída aos danos por umidade, os quais foram se tornando significativos já na época $\mathrm{E}_{5} \mathrm{~T}$ (sementes tratadas e analisadas em setembro, 90 dias antes da semeadura). Estes danos aumentaram em extensão, atingindo dessa forma regiões críticas das sementes, diminuindo o vigor e a viabilidade. Goulart (1998) considera que o tratamento fungicida não tem efeito sobre fatores como dano mecânico, deterioração por umidade, ataque de percevejo e armazenagem inadequada, que causam a redução da qualidade fisiológica das sementes. 
TABELA 6. Avaliação de danos por umidade (\%), pelo teste de tetrazólio, em sementes de soja da cultivar BR-16, produzidas na safra de 1999/2000, e armazenadas em Marechal Cândido Rondon, PR, tratadas com fungicida em diferentes épocas.

\begin{tabular}{cccc}
\hline & \multicolumn{2}{c}{ Tratamento } & \\
\cline { 2 - 3 } Época & \multicolumn{1}{c}{$\mathrm{T}_{0}$} & $\mathrm{~T}_{1}$ & $\mathrm{CV}(\%)$ \\
\hline $\mathrm{E}_{1} \mathrm{~T}$ & $1 \mathrm{Acd}$ & $1 \mathrm{Ac}$ & 18,09 \\
$\mathrm{E}_{2} \mathrm{~T}$ & $5 \mathrm{Abcd}$ & $8 \mathrm{Abc}$ & 21,19 \\
$\mathrm{E}_{3} \mathrm{~T}$ & $0 \mathrm{Ad}$ & $4 \mathrm{Aabc}$ & 17,71 \\
$\mathrm{E}_{4} \mathrm{~T}$ & $2 \mathrm{Abcd}$ & $7 \mathrm{Aabc}$ & 42,88 \\
$\mathrm{E}_{5} \mathrm{~T}$ & $13 \mathrm{Aab}$ & $10 \mathrm{Aabc}$ & 9,92 \\
$\mathrm{E}_{6} \mathrm{~T}$ & $3 \mathrm{Abcd}$ & $10 \mathrm{Aabc}$ & 44,05 \\
$\mathrm{E}_{7} \mathrm{~T}$ & $10 \mathrm{Aabc}$ & $14 \mathrm{Aab}$ & 33,19 \\
$\mathrm{E}_{8} \mathrm{~T}$ & $24 \mathrm{Aa}$ & $21 \mathrm{Aa}$ & 8,80 \\
$\mathrm{E}_{1} \mathrm{~A}$ & $24 \mathrm{Aa}$ & $21 \mathrm{Aa}$ & 12,64 \\
$\mathrm{E}_{2} \mathrm{~A}$ & $24 \mathrm{Aa}$ & $10 \mathrm{Aab}$ & 21,57 \\
$\mathrm{E}_{3} \mathrm{~A}$ & $24 \mathrm{Aa}$ & $11 \mathrm{Babc}$ & 22,78 \\
$\mathrm{E}_{4} \mathrm{~A}$ & $24 \mathrm{Aa}$ & $16 \mathrm{Bab}$ & 5,88 \\
$\mathrm{E}_{5} \mathrm{~A}$ & $24 \mathrm{Aa}$ & $12 \mathrm{Babc}$ & 19,48 \\
$\mathrm{E}_{6} \mathrm{~A}$ & $24 \mathrm{Aa}$ & $15 \mathrm{Aab}$ & 16,12 \\
$\mathrm{E}_{7} \mathrm{~A}$ & $24 \mathrm{Aa}$ & $16 \mathrm{Aab}$ & 15,41 \\
\hline $\mathrm{CV}(\%)$ & 4,90 & 28,24 & \\
\hline
\end{tabular}

$\mathrm{T}_{1}$ - sementes tratadas com Carbendazin + Thiram; $\mathrm{T}_{0}-$ sementes sem tratamento. Médias seguidas pela mesma letra maiúscula na linha e minúscula na coluna, não diferem entre si pelo teste de Tukey, a 5\% de probabilidade.

\section{CONCLUSÕES}

Os testes de germinação e de tetrazólio não foram eficientes em mostrar os benefícios, nem os efeitos prejudiciais causados pelo tratamento fungicida;

$\mathrm{O}$ tratamento fungicida conferiu melhor emergência em campo, evidenciando a proteção às sementes;

Sementes que permaneceram tratadas por período superior a quatro meses tiveram desempenho inferior comparativamente às sementes tratadas nas demais épocas.

\section{REFERÊNCIAS}

BRASIL. Ministério da Agricultura e Reforma Agrária. Regras para análise de sementes. Brasília: SNDA/DNDV/CLAV, 1992. $365 \mathrm{p}$.

BRASIL. Ministério da Agricultura e Reforma Agrária. Padrões estaduais de sementes. Brasília: EMBRAPA/SPSB, 1993. 47p.

CARVALHO, N. M.; JACINTO, C. M. R. Época do tratamento fungicida em sementes de soja. Científica, Jaboticabal, v.7, n.2, p. 261-265, 1979.

DELOUCHE, J. C.; BASKIN, C.C. Accelerated aging techniques for predicting the relative storability of seed lots. Seed Science and Technology, Zürich, v.1, n.2, p. 427-552, 1973.

DHINGRA, O. O. Prejuízos causados por microrganismos durante o armazenamento de sementes. Revista Brasileira de Sementes, Brasília, n.1, p. 139-145, 1985.

EMPRESA BRASILEIRA DE PESQUISA AGROPECUÁRIA Recomendações técnicas para a cultura da soja no Paraná 1999/ 2000. Londrina: EMBRAPA-CNPSo, 1999. 236p. (Documentos, 131).

FRANÇA NETO, J. B.; KRYZANOWSKI, F. C.; SILVA, W. R. O teste de tetrazólio em sementes de soja. Londrina: EMBRAPACNPSo, 1998. 72p. (Documentos, 116).

FRANÇA NETO, J. B.; HENNING, A. A. Qualidade fisiológica e sanitária de sementes de soja. Londrina: EMBRAPA-CNPSo, 1984. 39p. (Circular Técnica, 9).

GOULART, A. C. P. Fungos em sementes de soja: detecção e importância. Dourados: EMBRAPA-CPAO, 1997. 58p. (Documentos, 11).

GOULART, A. C. P. Tratamento de sementes de soja com fungicidas: recomendações técnicas. Dourados: EMBRAPACPAO, 1998. 32p. (Circular técnica, 8).

GOULART, A. C. P.; FIALHO, W. F. B.; FUJINO, M. T. Viabilidade técnica do tratamento de sementes de soja com fungicidas antes do armazenamento. Dourados: EMBRAPA-CPAO, 1999. 41p. (Boletim de Pesquisa, 2).

GOULART, A. C. P.; CASSETARI NETO, D. Efeito do ambiente de armazenamento e tratamento químico na germinação, vigor e sanidade de sementes de soja, Glycine $\max$ (L.) Merril, com alto índice de Phomopsis sp. Revista Brasileira de Sementes, Brasília,v.9, n.3, p. 91-102, 1987.

HENNING, A. A. Testes de sanidade de sementes de soja. In: SOAVE,J.; WETZEL, M. M. V. S.(Ed.). Patologia de sementes. Campinas: Fundação Cargil, 1987. p.441-453.

HENNING, A. A.; ZORATO, M. F. Efeito do tratamento de sementes de soja com fungicidas antes do armazenamento. Informativo ABRATES, Curitiba, v.7, n.1/2, p.160, 1997.

LUCCA FILHO, O. A.; NOGUEZ, M. A. D.; BAUDET, L. M. Comparação entre épocas de tratamento de sementes de soja. In: CONGRESSO BRASILEIRO DE SEMENTES, 3., 1983a, Campinas. Resumos... Brasília: ABRATES, 1983a, p. 149.

LUCCA FILHO, O. A.; NOGUEZ, M. A. D.; BAUDET, L. M. Efeitos do tratamento com fungicidas sobre a qualidade das sementes de soja sob condições ambientais. In: CONGRESSO BRASILEIRO DE SEMENTES, 3.,1983, Campinas. Resumos... Brasília: ABRATES, 1983b, p. 151.

MARCOS FILHO, J.; SOUZA, F. H. D. Conservação de sementes de soja tratadas com fungicidas. Anais da E.S.A. "Luiz de Queiroz", v. 40, p. 181-201, 1983.

MENTEN, J. O. M. Importância do tratamento de sementes. In: MENTEN, J. O. M. (Ed.). Patógenos em sementes: detecção, danos e controle químico. Piracicaba: ESALQ/FEALQ, 1991. p. 203-224.

NOVEMBRE, A. D. L. C.; MARCOS FILHO, J. Tratamento fungicida e conservação de sementes de feijão. Revista Brasileira de Sementes, Brasília, v. 13, n. 2, p. 105-113, 1991. 
OLIVEIRA, J. A.; VIEIRA, M. G. G. C.; VON PINHO, E. V. R.; CARVALHO, M. L. M. Comportamento de sementes de milho tratadas com fungicidas antes e após o armazenamento convencional. Revista Brasileira de Sementes, Brasília, v. 19, n. 2, p. 208-213, 1997.

PEREIRA, L. A. G.; COSTA, N. P.; ALMEIDA, M. R.; FRANÇA NETO, J. B.; GILIOLI, J. L.; HENNING, A. A. Tratamento de sementes de soja com fungicida e/ou antibiótico, sob condição de semeadura em solo com baixa disponibilidade hídrica. Revista Brasileira de Sementes, Brasília, v. 15, n. 2, p. 241-246, 1993.

TELLA, R.; LAGO, A. A.; ZINK, E. Efeitos de diversos níveis de umidade e tratamento fungicida, na longevidade de sementes de amendoim. Bragantia, Campinas, v. 35, n. 27, p. 335-342, 1976.
ZORATO, M. F. Influência de diferentes formulações de fungicidas e agentes veiculadores, na qualidade de sementes de soja armazenada. Londrina. 1998. 89f. Dissertação (Mestrado em Agronomia) - Universidade Estadual de Londrina, Londrina, 1998.

ZORATO, M. F.; HENNING, A. A. Viabilidade de utilização e influência de agentes veiculadores de fungicidas, aplicados em diferentes épocas de armazenamento, na qualidade de sementes de soja. Revista Brasileira de Sementes, Brasília, v 21, n 1, p. 208-215, 1999.

ZORATO, M. F.; HENNING, A. A. Influência de tratamentos fungicidas antecipados, aplicados em diferentes épocas de armazenamento, sobre a qualidade de sementes de soja. Revista Brasileira de Sementes, Brasília, v 23, n 2, p 236-244, 2001. 